\title{
Prevalence of primary dysmenorrhea and associated factors in adult women
}

\author{
Luana Brito dos Santos ${ }^{1}$ (), Isabelle Ribeiro Barbosa ${ }^{1}$ (), Thaissa Hamana de Macedo Dantas ${ }^{1}$ (i), \\ Camilla Medeiros Araujo ${ }^{2}$ (D), Jardelina Hermecina Dantas ${ }^{1}$ (ㄹ, Caroline Wanderley Souto Ferreira $^{2}$ (D), \\ Saionara Maria Aires da Câmara ${ }^{1}$ (1), Diego Dantas ${ }^{1,2 *}$ (1)
}

\section{SUMMARY}

OBJECTIVE: This study aimed to assess the prevalence and factors associated with primary dysmenorrhea in a sample of adult women. METHODS: A cross-sectional study was carried out with women aged between 19 and 49 years from a city of northeastern Brazil. Sociodemographic, gynecological, and obstetric variables were assessed by questionnaires and interviews. Dysmenorrhea was measured by self-report, and the Numerical Pain Rating Scale measured the intensity of pain. Statistical analyses included $\chi^{2}$ test, ANOVA, and logistic regression.

RESULTS: The average age was 33.2 \pm 9.1 years and the prevalence of primary dysmenorrhea was $56 \%$ for the whole sample. The average duration of symptoms was $2.7 \pm 1.8$ days and the mean intensity was $6.1 \pm 2.6$. The previous cesarean section was associated with a higher rate of primary dysmenorrhea ( $P R=2.33 ; 95 \% \mathrm{Cl} 1.11-4.90)$ when considering the whole sample. Women who aged 25-39 years and are insufficiently active had higher rates of primary dysmenorrhea ( $P R=5.24 ; 95 \% \mathrm{Cl} 1.08-27.31$ ).

CONCLUSION: Primary dysmenorrhea has a high prevalence in young adults, adults, and middle-aged women. Cesarean section and being physically inactive was associated with increased rates of dysmenorrhea among adult women.

KEYWORDS: Cross-sectional studies. Dysmenorrhea. Epidemiology. Prevalence. Women's health.

\section{INTRODUCTION}

Dysmenorrhea is defined as colic pain in the hypogastrium that occurs during menstruation and other symptoms like sweating, headaches, nausea, vomit, diarrhea, and tremors may occur associated ${ }^{1}$. Dysmenorrhea is classified into primary (menstrual pain without organic disease) or secondary (menstrual pain associated with another preexistent disease, e.g., endometriosis $)^{1,2}$.

The prevalence of primary dysmenorrhea (PD) is well studied among teenagers and youngers in different countries and it ranges from 16 to $91 \%{ }^{3,4}$. Several risk factors are established in the literature and the casuistic of PD may involve social, demographic, behavioral, gynecological, and reproductive issues ${ }^{1,4,5}$.

As the majority of studies about PD were conducted with students and teenagers, the generalization of these evidence for women of all adult age, including middle-aged women, are limited and needs more clarification ${ }^{4}$. Besides that, it is estimated that the prevalence of PD is even higher since many women associate dysmenorrhea as a normal menstrual cycle pain and do not seek medical assistance for this condition ${ }^{1}$.
The perception and coping of pain related to PD vary from the women's context. Then, issues related to work, social roles, and women's empowerment can modify these perception among different age groups ${ }^{6}$. This fact reinforces the relevance to know the prevalence of PD and associated factors in the different stages of a woman's adult life and not just in adolescence.

Moreover, PD represents relevant cause of school and work absence, negatively affects academic performance, productivity, daily life activities, and quality of life of these women ${ }^{4,7}$. Then, knowing the prevalence and associated factors in the different ages of woman's life may allow to intervene and minimize the impacts of dysmenorrhea on the lives of these women ${ }^{8}$. Therefore, this study aimed to assess the prevalence and associated factors to PD in adult women (19-49 years of age).

\section{METHODS}

A cross-sectional, community-based study was conducted from December 2015 to November 2016, in the municipality

\footnotetext{
${ }^{1}$ Universidade Federal do Rio Grande do Norte, Faculdade de Ciências da Saúde do Trairi - Santa Cruz (RN), Brazil.

¿Universidade Federal de Pernambuco, Departamento de Fisioterapia - Recife (PE), Brazil.

*Corresponding author: diego.sdantas@ufpe.br

Conflicts of interest: the authors declare there is no conflicts of interest. Funding: none.

Received on September 11, 2021. Accepted on November 01, 2021.
} 
of Santa Cruz, Rio Grande do Norte, Brazil. The Ethical and Research Committee of Federal University of Rio Grande do Norte approved the research protocol under number CAAE 49237315.9.0000.5568. The study followed the Declaration of Helsinki and all the participants signed the informed consent form.

The sample size was determined using the prevalence of $67.5 \%$ of dysmenorrhea, power of $90 \%$, and a nonresponse rate of 5\%, resulting in a sample of 195 women. This prevalence was obtained in a previous pilot study with 30 women following the same inclusion criteria (data not shown).

Women were proportionally recruited from among the six services of primary healthcare services of the city. Women were approached for convenience while accompanying a relative or waiting for an appointment for themselves. Previously trained interviewers conducted the interviews in a silent, private place of the health care services.

Women, who aged 19-49 years, with the regular menstrual flow in the last 3 months, and who accepted to participate in the study, were included. Exclusion criteria included women who reported a medical diagnosis of endometriosis, adenomyosis, uterine myoma, or pelvic inflammatory disease; in hormone replacement therapy; or had a history of hysterectomy.

Primary dysmenorrhea was assessed by self-reporting of colic-type pain in the lower abdomen associated with menstruation in the last 3 months ${ }^{4}$. The Numeric Pain Rating Scale (NPRS) assesses the intensity of pain $(0-10)^{9}$. The duration of pain was expressed in days.

Independent variables were obtained by conducting interview and include age (grouped into young adults: 19-24 years, adults: $25-39$ years, and middle-aged women: $40-49$ years); family income ( $\leq 1$ minimum wage or more); religion; age of menarche ( $\leq 11$ years old or $\geq 12$ ); use of any contraceptive method; menstrual cycle length dichotomized into 28-35 days and ( $<28$ or $>35$ days); and number of children, cesarean section, and vaginal delivery.

Constipation was determined according to ROMA III criteria $^{10}$ and the sexual dysfunction was defined as a score $<60$ points in the Female Sexual Quotient Questionnaire ${ }^{11}$. The presence and type of urinary incontinence was determined by conducting interview following the recommendations of International Continence Society ${ }^{12}$. The short-form of International Physical Activity Questionnaire (IPAQ) evaluated the level of physical activity, and <600 METs-minute/week indicated insufficiently active women ${ }^{13}$.

Data analyzes were conducted by the software SPSS, version 22. The Kolmogorov-Smirnov test confirms the normal distribution of continuous data. We expressed categorical data by absolute and relative frequencies, and continuous data through mean and standard deviations.

Prevalence of dysmenorrhea was calculated for overall and for age groups. ANOVA compared the variance among groups to intensity and duration of pain. Binary logistic regression was performed and the variables with $\mathrm{p}<0.20$ were included in the multivariate logistic regression. Statistical significance was defined as $\mathrm{p}<0.05$.

\section{RESULTS}

Initially, we invited 211 women to participate. Of those, 16 were excluded for having a history of hysterectomy $(n=7)$ or being pregnant $(n=9)$, resulting in 195 women included in the study with a mean age of 33.16 \pm 9.06 years old. Sociodemographic and clinical profile is shown in Table 1.

The prevalence of $\mathrm{PD}$ was $55.9 \%$ in all samples, and $50 \%$ in young adults, $55 \%$ in adults, and $61 \%$ in middle-aged women. Pain intensity and duration of complaints did not differ among groups (Table 2).

The results of univariate and multivariate logistic regression are shown in Table 3. Having at least one cesarean section was associated with 2.33 times risk of having PD among women aged 19-49 years. Among the adult women, who were insufficiently physically active, it was associated with 5.42 times risk of having PD.

\section{DISCUSSION}

Primary dysmenorrhea is characterized by uterine hypercontractility provoked by the overproduction of prostaglandins in the endometrium, leading to uterine muscle ischemia, hypoxia, and, subsequently, pain ${ }^{14}$. Although PD is often a gynecological complaint, it is underdiagnosed, undertreated, and even undervalued by women themselves ${ }^{14}$. Our findings show that PS has a high prevalence throughout a woman's adulthood, contradicting evidence that suggests a decrease in pain after pregnancies ${ }^{3}$. However, it was observed that risk factors may differ according to the progression of the life cycle and classical factors involved in physiology of PD were not associated with contraceptive methods, menarche age, and having children ${ }^{4,14}$.

In our study, cesarean section was associated with more cases of PD. It is a warning sign because the cesarean is the most common type of operation performed on women, and women who had undergone multiple cesarean sections can progress with cesarean scar defects ${ }^{15}$. The cesarean scar defect 
Table 1. Social demographic and clinical profile of sample grouped by age $(n=195)$.

\begin{tabular}{|c|c|c|c|}
\hline \multirow{3}{*}{ Variables } & \multicolumn{3}{|c|}{ Women } \\
\hline & Young adults $(n=40)$ & Adults $(n=96)$ & Middle-aged women $(n=59)$ \\
\hline & \multicolumn{3}{|c|}{$\%$} \\
\hline \multicolumn{4}{|l|}{ Family income* } \\
\hline$\leq 1$ minimum wage & 80.0 & 79.2 & 79.7 \\
\hline >1 minimum wage & 20.0 & 20.8 & 20.3 \\
\hline Profess some religion & 77.5 & 85.4 & 88.1 \\
\hline Insufficiently physically active & 17.5 & 21.9 & 13.6 \\
\hline \multicolumn{4}{|l|}{ Age of menarche } \\
\hline$\leq 11$ years old & 17.5 & 17.7 & 22.0 \\
\hline$\geq 12$ years old & 82.5 & 82.3 & 78.0 \\
\hline Use any contraceptive method & 60.0 & 49.5 & 55.2 \\
\hline \multicolumn{4}{|l|}{ Menstrual cycle length $(n=172)$} \\
\hline 28-35 days & 68.6 & 76.2 & 71.7 \\
\hline$<28$ days or $>35$ days & 31.4 & 23.8 & 28.3 \\
\hline Constipation & 37.5 & 29.2 & 44.1 \\
\hline Urinary incontinence & 15 & 16.7 & 30.5 \\
\hline Stress urinary incontinence & 7.5 & 6.3 & 16.9 \\
\hline Urgency urinary incontinence & 2.5 & 4.2 & 6.8 \\
\hline Mixed urinary incontinence & 5.0 & 6.3 & 6.8 \\
\hline Sexual dysfunction & 27.5 & 36.7 & 40.0 \\
\hline Have children & 75.0 & 82.3 & 81.4 \\
\hline$\geq 1$ Cesarean section $(n=146)$ & 70.0 & 66.7 & 61.0 \\
\hline$\geq 1$ Vaginal delivery $(n=146)$ & 67.5 & 76.0 & 69.5 \\
\hline
\end{tabular}

${ }^{*}$ In 2016, the value of a minimum wage in Brazil was $\mathrm{R} \$ 880.00$.

Table 2. Comparative analyses of mean intensity and duration of pain during the menstrual cycle among young adult, adult, and middle-aged women $(n=109)$.

\begin{tabular}{l|c|c} 
& $\begin{array}{c}\text { Intensity of pain (NPRS) } \\
\text { Mean } \pm \text { SD }\end{array}$ & $\begin{array}{c}\text { Duration of pain (days) } \\
\text { Mean } \pm \text { SD }\end{array}$ \\
\hline Total sample & $6.14 \pm 2.55$ & $2.68 \pm 1.83$ \\
\hline Young adults & $6.70 \pm 2.58$ & $2.80 \pm 1.61$ \\
\hline Adults & $5.87 \pm 2.55$ & $2.40 \pm 1.57$ \\
\hline Middle-aged womens & $6.22 \pm 2.56$ & $3.03 \pm 2.22$ \\
\hline ANOVA (p-value) & 0.454 & 0.265 \\
\hline Young adults×adults (p-value)* & 0.433 & 0.477 \\
\hline Young adults×middle-aged women (p-value)* & 0.782 & 0.895 \\
\hline Adultxmiddle-aged women (p-value)* & 0.798 & 0.247 \\
\hline
\end{tabular}

${ }^{*}$ p-value was calculated by the post hoc Tukey test. NPRS: Numeric Pain Rating Scale. 


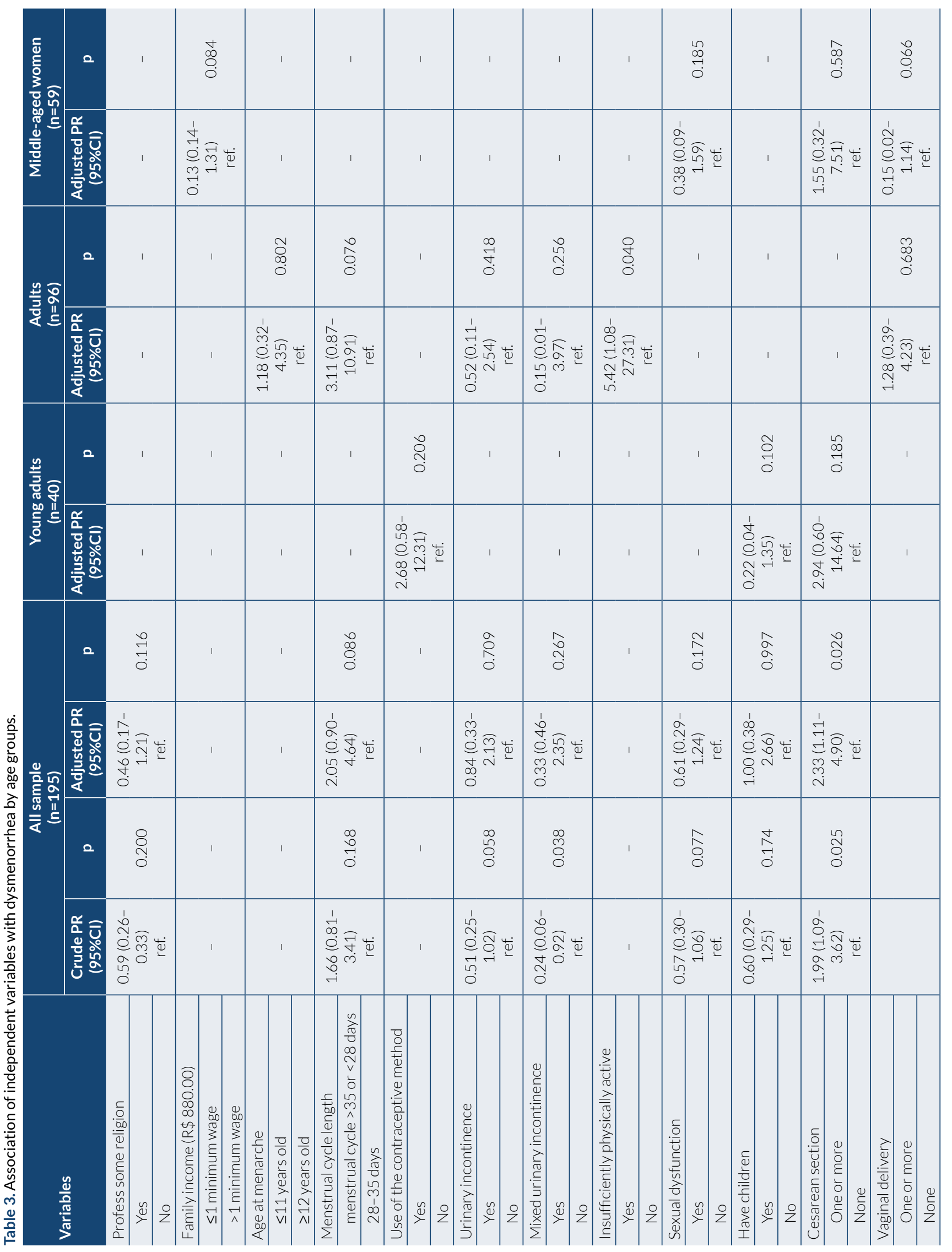


when symptomatic is often related to dysmenorrhea and chronic pelvic pain ${ }^{16,17}$. In this context, the nerve section, inadvertent nerve ligation of fibrous scarring, and myofascial syndrome result in menstrual and chronic pelvic pain ${ }^{17,18}$.

Physical inactivity was associated with a higher frequency of PD. The physical activity has positive effects on stress, prostaglandin levels, and blood circulation, resulting in decreased pain and the prevalence of dysmenorrhea ${ }^{19}$. Then, women who do not perform exercise do not receive this benefit of endogenous opioids ${ }^{20}$. Therefore, it is one more motif to encourage women to remain physically active.

Our data are innovative by assessing PD in a sample containing older adults than other studies available in the Brazilian population. Despite this, the generalization of data should be made with caution because our data were collected in a small town, and the prevalence and associated factors to dysmenorrhea in women who live in large cities can be different.

As a limitation of the study, we can mention the use of a convenience sample, the cross-sectional design that prevents the establishment of cause-and-effect relationships, and the collection of data through interviews. For future studies, more robust methodological designs that include clinical assessment

\section{REFERENCES}

1. Bernardi M, Lazzeri L, Perelli F, Reis FM, Petraglia F. Dysmenorrhea and related disorders. F1000Res. 2017;6:1645. https://doi. org/10.12688/f1000research.11682.1

2. Urbano-Ruiz A, Soares Jr JM, Rossi AGZ, Louback RM, Hummel FV, Baracat EC. Female pelvic pain: a case report of mucocele of the appendix. Eur J Gynaecol Oncol. 2011;32(6):672-3. PMID: 22335033

3. Ju H, Jones M, Mishra G. The prevalence and risk factors of dysmenorrhea. Epidemiol Rev. 2014;36(1):104-13. https://doi. org/10.1093/epirev/mxt009

4. Burnett M, Lemyre M. No. 345-Primary Dysmenorrhea Consensus Guideline. J Obstet Gynaecol Can. 2017;39(7):585-95. https:// doi.org/10.1016/j.jogc.2016.12.023

5. Nicolau SM, Soares JM, Schor E, Gonçalves WJ, Freitas V, Baracat EC. Schistosoma mansoni pseudo-cyst as a cause of chronic pelvic pain. J Obstet Gynaecol Res. 2003;29(6):392-4. https://doi. org/10.1111/j.1341-8076.2003.00134.x

6. Samulowitz A, Gremyr I, Eriksson E, Hensing G. "Brave men" and "emotional women": a theory-guided literature review on gender bias in health care and gendered norms towards patients with chronic pain. Pain Res Manag. 2018;2018:6358624. https://doi. org/10.1155/2018/6358624

7. Hashim RT, Alkhalifah SS, Alsalman AA, Alfaris DM, Alhussaini MA, Qasim RS, et al. Prevalence of primary dysmenorrhea and its effect on the quality of life amongst female medical students at King Saud University, Riyadh, Saudi Arabia. Saudi Med J. 2020;41(3):283-9. https://doi.org/10.15537/smj.2020.3.24988 are recommended to better establish the relationships found in this study.

\section{CONCLUSION}

There was a high prevalence of PD with rates above $50 \%$ in adult women of all ages. In this study, only cesarean section and being physically inactive was associated with increased rates of dysmenorrhea among adult women.

\section{AUTHORS' CONTRIBUTIONS}

LBS: Conceptualization, Methodology, Formal Analysis, Investigation, Data curation, Writing - original draft, Writing review \& editing. IRB, THMD, CMA, JHD: Conceptualization, Methodology, Formal Analysis, Data curation, Writing - original draft, Writing - review \& editing. CWSF: Formal Analysis, Data curation, Writing - original draft, Writing - review \& editing. SMAC: Formal Analysis, Data curation, Writing - original draft, Writing - review \& editing, Funding acquisition. DD: Conceptualization, Methodology, Formal Analysis, Data curation, Writing - original draft, Writing - review \& editing, Project administration, Funding acquisition.

8. Mu J, Wang Q, Dun W, Yang J, Wang K, Zhang M, et al. The effects of long-term menstrual pain on pain empathy in women with primary dysmenorrhea. Pain. 2021;162(7):2051-9. https://doi. org/10.1097/j.pain.0000000000002205

9. Boonstra AM, Stewart RE, Köke AJ, Oosterwijk RFA, Swaan JL, Schreurs KMG, et al. Cut-off points for mild, moderate, and severe pain on the numeric rating scale for pain in patients with chronic musculoskeletal pain: variability and influence of sex and catastrophizing. Front Psychol. 2016;7(Sep):1466. https://doi. org/10.3389/fpsyg.2016.01466

10. Dantas AAG, Barbosa IR, Castro SS, Ferreira CWS, Camara SMA, Dantas DS. Prevalence and factors associated with constipation in premenopausal women: a community-based study. Arq Gastroenterol. 2020;57(2):188-92. https://doi.org/10.1590/ S0004-2803.202000000-36

11. Abdo CHN. Quociente sexual feminino: um questionário brasileiro para avaliar a atividade sexual da mulher. Diagn Trat. 2009;14(2):89-1.

12. Milsom I, Altman D, Cartwright R, Lapitan MC, Nelson R, Sillén $\mathrm{U}$, et al. Epidemiology of urinary incontinence (UI) and other lower urinary tract symptoms (LUTS), pelvic organ prolapse (POP) and anal incontinence (AI). In: Abrams P, Cardozo L, Khoury S, Wein AJ, editors. Incontinence: 5th International Consultation on Incontinence. 5th ed. Paris: ICUD-EAU; 2013. p. 15-107. https://www.ics.org/Publications/ICI_5/INCONTINENCE.pdf.

13. Ahmad MH, Salleh R, Mohamad Nor NS, Baharuddin A, Rodzlan Hasani WS, Omar A, et al. Comparison between self-reported physical activity (IPAQ-SF) and pedometer among overweight and obese women in the MyBFF@home study. BMC Womens Health. 2018;18(Suppl 1):100.https://doi.org/10.1186/s12905-018-0599-8 
14. Guimarães I, Póvoa AM. Primary dysmenorrhea: assessment and treatment. Rev Bras Ginecol Obstet. 2020;42(8):501-7. https:// doi.org/10.1055/s-0040-1712131

15. Tulandi T, Cohen A. Emerging manifestations of cesarean scar defect in reproductive-aged women. J Minim Invasive Gynecol. 2016;23(6):893-902. https://doi.org/10.1016/j. jmig.2016.06.020

16. Deng J, Li S, Peng Y, Chen Z, Wang C, Fan Z, et al. Chinese herbal medicine for previous cesarean scar defect: a protocol for systematic review and meta-analysis. Medicine (Baltimore). 2020;99(50):e23630. https://doi.org/10.1097/ MD.0000000000023630

17. Nogueira AA, e Silva JCR, Poli Neto OB. The potential of cesarean section as a causative factor of chronic pelvic pain. Rev Bras Ginecol Obstet. 2016;38(2):53-5. https://doi. org/10.1055/s-0036-1571850

18. Li R, LiB, Kreher DA, Benjamin AR, Gubbels A, Smith SM. Association between dysmenorrhea and chronic pain: a systematic review and meta-analysis of population-based studies. Am J Obstet Gynecol. 2020;223(3):350-71.https://doi.org/10.1016/j.ajog.2020.03.002

19. Prazeres LMA, Brito RG, Ramos ES. Regular physical exercise, sedentarism and characteristics of dismenorrhea and premenstrual syndrome. Fisioter Mov. 2018;31. https://doi.org/10.1590/19805918.031.AO18

20. Carroquino-Garcia P, Jiménez-Rejano JJ, Medrano-Sanchez E, Casa-Almeida M, Diaz-Mohedo E, Suarez-Serrano C. Therapeutic exercise in the treatment of primary dysmenorrhea: a systematic review and meta-analysis. Phys Ther. 2019;99(10):1371-80. https://doi.org/10.1093/ptj/pzz101 